\title{
Inventário de Depressão de Beck - BDI: validação fatorial para mulheres com câncer ${ }^{1}$
}

\author{
Rita de Cássia Gandini² \\ Maria do Carmo Fernandes Martins \\ Marjorie de Paula Ribeiro \\ Daniela Torres Gonçalves Santos
}

\begin{abstract}
Resumo
Este estudo teve por objetivo validar fatorialmente o Inventário de Depressão de Beck - BDI para uma amostra de 208 mulheres com câncer. A validação fatorial do BDI foi realizada por meio da análise fatorial, método de extração dos componentes principais e rotação oblimin, tendo sido identificadas duas soluções fatoriais, uma bifatorial sendo F1 composto por 8 itens $(\alpha=0,82)$ denominado "visão negativa de si mesmo", explicando 32,4\% da variância e F2 com 5 itens $(\alpha=0,77)$ denominado "função física", explicando $8 \%$ da variância - e outra unifatorial com 13 itens, com fidedignidade altamente satisfatória $(\alpha=0,86)$ denominada "depressão", explicando 38\% da variância total.
\end{abstract}

Palavras-chave: Depressão; Câncer; Inventário de Depressão de Beck; Análise fatorial; BDI.

\section{Beck Depression Inventory - BDI: Factorial analysis for women with cancer}

\begin{abstract}
This study refers to the factor analysis of the Beck Depression Inventory - BDI in a population composed by 208 women with cancer. The extraction method was principal components and the rotation procedure was oblimin. The BDI factorial analysis was done through the oblimin rotation, finding two solutions possibilities. One with two factors solution - the first one with 8 items $(\alpha=0,82)$, called "negative view of self", explaining 32,4\% of the variance and the second factor with 5 items $(\alpha=0,77)$, called "physical function", explaining $8 \%$ of the variance and other with one factor solution with 13 items, with really satisfactory reliability of 0,86 , called "depression" and explaining $38 \%$ of the total variance.
\end{abstract}

Keywords: Depression; Cancer; Beck Depression Inventory; Factor analysis; BDI.

\section{Introdução}

Com o aumento do número de casos de câncer de mama, a preocupação dos pesquisadores tem se voltado cada vez mais para o diagnóstico psicológico a ser realizado de forma rápida e eficaz para, a partir daí, realizar uma intervenção imediata, focalizada nos sintomas apresentados pela paciente, minimizando seu sofrimento. Para tal, é necessário avaliar precocemente a paciente quando a mesma vem para a primeira consulta oncológica com forte hipótese de ser portadora da doença.

O estudo de validação fatorial da Escala BSI "Brief Symptom Inventory" para amostra mineira (Martins, Gandini \& Costa, 2000), que avalia sintomas físicos, ansiedade, depressão e sinais de depressão com idéias paranóides, evidenciou depressão nos sujeitos, pacientes com câncer de mama, o que causou grande preocupação e curiosidade científica acerca dos índices de depressão em mulheres com outros tipos de câncer. Em trabalho anterior, Pellegrino-Rosa, Gandini, Rosa, Wutke e Simon (1991) haviam detectado traços de melancolia, que diferenciavam a população com câncer de mama da que apresentava depressão clássica, assim como daquela que não apresentava depressão.

A depressão, assim como o câncer, tem sido considerada doença que se apresenta como grave problema de saúde pública, estando o câncer em segundo lugar nas estatísticas de causa de morte por doença no mundo (Horimoto, Ayache \& Souza, 2005; INCA, 2005). Com esses dados, pareceu necessário e iminente aprofundar o conhecimento acerca da avaliação da depressão para a população feminina com câncer. $O$ Inventário de Depressão de Beck - BDI é um instrumento que atende ao propósito de avaliar a depressão, mas não foi localizado na literatura nacional nenhum estudo sobre sua validade para amostras brasileiras específicas como a de pacientes com

\footnotetext{
${ }^{1}$ Apoio institucional: CNPq (bolsa de PIBIC/UFU)

${ }^{2}$ Endereço para correspondência:

Rua Paraíba, 1.795 apto 22 - Condomínio Vitória Régia - Bairro Marta Helena - 38402-014 - Uberlândia-MG

E-mail: rcgandini@uol.com.br
} 
câncer.

Por isso, a proposta deste estudo foi validar fatorialmente a Escala - BDI em pacientes com câncer a fim de identificar a estrutura fatorial da depressão em mulheres com tal patologia. No momento do projeto, desenvolvimento e confecção do relatório final deste estudo (Gandini, Martins, Ribeiro \& Santos, 2001), entregue ao órgão de fomento - $\mathrm{CNPq}$, não havia sido publicado o Manual da versão em português das Escalas BECK (Cunha, 2001). Supõe-se que ambos os trabalhos tenham sido realizados concomitantemente.

O Inventário de Depressão de Beck - BDI foi desenvolvido originariamente por Beck, Ward, Mendelson, Mock e Erbaugh (1961). Trata-se de uma escala de autorelato, para levantamento da intensidade dos sintomas depressivos (Beck \& Steer, 1993; Rosa, Malandrin, Leite \& Silva, 1986), composta por 21 itens e estimativas de fidedignidade estabelecidas a partir de seis amostras psiquiátricas (Beck \& Steer, 1993) que variaram entre 0,79 e 0,90. Segundo Cunha (2001), é um instrumento particularmente adequado para uso com pacientes psiquiátricos que, porém, tem sido amplamente usado na clínica e em pesquisa com pacientes não psiquiátricos e na população geral. Em pacientes com câncer, o BDI foi utilizado em adolescentes (Berard \& Boermester, 1998), pacientes com câncer na cabeça e pescoço sob tratamento radioterápico (Chawla e cols., 1999), pacientes com câncer de pâncreas (Kelsen e cols., 1995) e em mulheres com câncer de mama, avaliando os efeitos da psicoterapia na depressão apresentada por elas (Marchioro e cols., 1996).

Diversos estudos de validação do BDI têm sido realizados. Estudos de validade de critério para pacientes mexicanos com artrite reumatóide (Suarez-Mendoza, Caballero-Uribe, Ortega-Soto \& Marquez-Marin, 1997); de validade discriminante em adolescentes com depressão clínica (Bennet e cols., 1997); de validade convergente em pacientes com pânico, com depressão e em estudantes universitários (Gorenstein \& Andrade, 1996); e de validade de construto com pacientes psiquiátricos (Richter e cols., 1997). Todos esses trabalhos padronizaram e demonstraram a utilidade do BDI nessas populações, para diferentes fins.

Entretanto, foram poucos os estudos de validação fatorial do BDI encontrados na literatura internacional e no Brasil. Entre eles, destaca-se o estudo de validade de construto do BDI para população com depressão unipolar - DSM-III (Schotte, Maes, Cluydts, Doncker \& Cosyns, 1997), que, além da validação convergente, realizou a análise fatorial. Os autores mostraram duas possibilidades de interpretação, utilizando dois ou três fatores. Como o índice de fidedignidade do terceiro fator não era satisfatório $(\alpha=0,54)$ e o conteúdo do primeiro fator era heterogêneo, os autores optaram pela solução de dois fatores, com $\alpha$ de 0,86 e 0,83 , respectivamente, reunindo 16 itens. A subescala somática, o segundo fator, apresentou resultado significantemente diferente em relação ao gênero, pois pacientes mulheres obtiveram escores mais altos.

Morley, Williams e Black (2002) avaliaram a depressão de 1.947 pacientes com dor crônica e testaram a estrutura fatorial do BDI. Identificaram dois fatores fortes, que chamaram "visão negativa do eu" e "função física e somática". Submetendo os mesmos dados à análise fatorial confirmatória, identificaram a mesma estrutura. Em ambas as análises retiveram 13 dos 21 itens originais do BDI.

Os resultados da análise fatorial do BDI em outro estudo (Grunebaum e cols., 2005) com pacientes psiquiátricos, diagnosticados com transtorno depressivo maior, indicaram uma estrutura composta por três fatores: F1 = depressão subjetiva, F2 = auto-acusação e F3 = queixas somáticas. Para a extração dos fatores foi utilizada a rotação não ortogonal promax, e os autores justificaram sua utilização pela sobreposição dos componentes da depressão, sem, todavia, testar a correlação entre os fatores.

No Brasil, um dos estudos de validação fatorial do BDI foi realizado por Gorenstein e Andrade (1998) com 1.080 estudantes universitários da cidade de São Paulo. Utilizando rotação varimax, as autoras identificaram três fatores para a amostra total. $O$ fator 1 representando a dimensão de autodepreciação, o fator 2, cognição-afeto e o fator 3, a dimensão somática (ver Tabela 1). Os coeficientes de fidedignidade (alfa de Cronbach) foram de 0,76; $0,77 \mathrm{e}$ 0,66 , e cada fator explicou 28,3\%; 6,4\% e 6,1\%, respectivamente, da variância total. As autoras subdividiram a amostra quanto ao sexo e realizaram nova análise fatorial para cada subgrupo. Para ambas, encontraram dois fatores. Os itens do fator 3 da amostra geral foram retidos nos dois fatores das amostras divididas por gênero, evidenciando estruturas diferentes da identificada na amostra geral. Pela análise desses fatores, Gorenstein e Andrade (1998) concluíram que, para as mulheres, a auto-depreciação associou-se mais intimamente à experiência depressiva do que para os homens.

No mesmo trabalho, Gorenstein e Andrade (1998) testaram ainda a estrutura fatorial do BDI numa amostra de 374 adolescentes do ensino fundamental e médio, com idade média de 14,7 anos ( $\mathrm{DP}=2,1)$. Identificaram dois fatores, para a amostra total, responsáveis por $21,5 \%$ e $7,3 \%$ da variância, respectivamente. $\mathrm{O}$ fator 1 reuniu 10 itens $\operatorname{com} \alpha=0,74$, representando a dimensão cognição-afeto. $\mathrm{O}$ fator 2 , com 6 itens e $\alpha=0,53$, representou a dimensão somática (ver Tabela 1).

Um outro estudo encontrado no Brasil foi o realizado por Pellegrino-Rosa (1997), com 153 idosos 
saudáveis com idades entre 50 e 77 anos. Foi utilizada a rotação varimax, e retidos os itens com valores próprios maiores que 1. Os resultados apontaram a retenção de um fator com 5 itens, que explicou $51 \%$ da variância total (ver Tabela 2).

As validações fatoriais feitas no Brasil por Cunha e cols. (1995, citado por Cunha, 2001), para uma amostra de 97 pacientes com dependência de álcool, e para outra, de 204 pacientes com episódio de depressão maior sem co-morbidade utilizaram rotação varimax $\mathrm{e}$ identificaram três fatores. A autora não apresentou a composição dos fatores com suas respectivas cargas nem o índice de fidedignidade em ambas as amostras.

A validação do BDI para uma comunidade chinesa que vive no Brasil e que fala português foi realizada por Wang, Andrade e Gorenstein (2005) com 208 sujeitos, utilizando-se da rotação varimax. Este estudo encontrou para amostra total uma solução de dois fatores com $\alpha=0,79$ e 0,67 , respectivamente, explicando 31,45\% da variância total (ver Tabela 2). Tanto para a amostra masculina quanto para a amostra feminina também foram encontrados dois fatores, abrangendo a dimensão cognitivo-afetiva e a dimensão somática, com $\alpha=0,81$ e 0,70 para a amostra masculina e $\alpha=0,76$ e 0,73 para a feminina.

Os trabalhos brasileiros relatados anteriormente identificaram diferentes estruturas fatoriais que variaram de um a três fatores. Foram também utilizados métodos de rotação diferentes, tanto nos estudos internacionais quanto nos brasileiros. Havia então dois desafios a serem enfrentados: validar o inventário para pacientes com câncer e optar pela rotação mais adequada, apontada por testes específicos para tal.

\section{Objetivos}

Os objetivos deste estudo foram validar fatorialmente o Inventário de Depressão de Beck -BDI e estabelecer seus índices de fidedignidade.

\section{Método}

\section{Participantes}

A amostra foi constituída de 208 mulheres, tanto pacientes com câncer de mama (46\%) que estavam em tratamento no serviço de Mastologia Maligna do Hospital das Clínicas da UFU quanto pacientes portadoras de câncer em geral (54\%), atendidas no Hospital do Câncer de Uberlândia. A idade variou entre 20 e 87 anos, com média de 53 anos $(\mathrm{DP}=12,5)$. A maioria $(51 \%)$ dos participantes possuía escolaridade de $1^{\text {a }}$ a $4^{\text {a }}$ série do ensino fundamental;
$14 \%$ eram analfabetas; $18 \%$ tinham de $5^{a}$ a $8^{a}$ série; $3 \%$ possuíam ensino médio incompleto; $9 \%$ ensino médio completo; $0,5 \%$ nível superior incompleto e $4 \%$ nível superior completo.

As profissões dos participantes concentraramse em atividades desenvolvidas no lar (52\%), empregadas domésticas $(13 \%)$ e costureiras $(11 \%)$. As demais $24 \%$ das pacientes desempenhavam outras 31 profissões. A maioria era casada ou amasiada (54\%); $19 \%$ eram viúvas; $16 \%$, solteiras e $11 \%$ separadas/divorciadas.

Procedimento de adaptação semântica e hierarquização das respostas do Inventário de Depressão de Beck

Com base na forma original do BDI, algumas expressões desses conjuntos foram adaptadas semanticamente por apresentarem dificuldade de compreensão por parte da amostra, como nos itens que utilizavam termos temporais (a palavra "usual" foi mudada por "como de costume", por exemplo). Os 21 conjuntos de expressões originais em português foram apresentados a 20 sujeitos representantes da amostra para verificação da clareza e compreensão dos conteúdos. Os conjuntos das respostas aos itens (escalas de respostas) foram ainda reorganizados de modo que passaram a ser hierarquizados em ordem crescente, ou seja, variando da ausência à presença forte de sintomatologia depressiva.

\section{Procedimento de coleta}

As pacientes tiveram o direito de escolher participar ou não da investigação e suas identidades foram preservadas. Em razão da baixa escolaridade das pessoas que compuseram a amostra, os questionários foram aplicados verbal e individualmente por pesquisadoras treinadas.

\section{Procedimento de análise dos dados}

Os conjuntos de opções de respostas referentes aos sintomas ou atitudes de depressão avaliados pelo Beck, em cerca de metade do questionário original, possuíam 5 opções de respostas e, na outra metade, somente 4 opções. Por isso, ao codificar os dados, aqueles conjuntos com 5 opções foram transformados em 4, tendo sido reunidas opções semanticamente muito próximas, como, por exemplo, "não consigo satisfação em mais nada" e "estou insatisfeito com tudo" de modo a uniformizar a escala de respostas, possibilitando a utilização da análise fatorial. Os dados foram, então, codificados no SPSS (Statistical Package of Social Sciences) e submetidos à análise fatorial com método de extração dos componentes principais (PC) e rotação oblimin, devido 
à interdependência dos fatores. Foram ainda calculados os alfas de Cronbach para todos os fatores.

\section{Resultados}

\section{Validação fatorial do BDI}

As respostas dos 208 participantes foram codificadas e analisadas no SPSS. A análise da fatorabilidade da amostra foi feita através do KMO (Índice de Kaiser, Meyer, Olkin), que revelou índice excelente $(0,90)$, segundo os critérios de Tabachnick e Fidell (2001). A interdependência entre os fatores foi verificada por meio da matriz de correlação entre os fatores, evidenciada nos resultados da rotação oblimin. A correlação foi significante, negativa e alta $(r=-0,42)$, o que revelou forte interdependência entre os dois fatores. Por isso foi mantida a rotação oblimin.

Os critérios utilizados para a seleção dos fatores foram valores próprios $\geq 1,5 \mathrm{e}$, para os itens, cargas $\geq 0,35$. A análise reteve, inicialmente, 2 fatores que explicaram $40 \%$ da variância total. Um exame do scree plot apontava a existência de 2 fatores.
O fator 1 "visão negativa de si mesmo" reuniu 8 itens: tristeza, pessimismo, sentimento de fracasso, culpa, autodepreciação, auto-acusação, idéias suicidas, mudança na imagem corporal, revelou alfa de Cronbach de 0,82 e explicou $32,4 \%$ do total da variância. O fator 2 "função física" reuniu 5 itens: dificuldade no trabalho, insônia, fatigabilidade, perda de apetite, perda da libido; apresentando um $\alpha=0,77$, e explicou $8 \%$ da variância total. Um item (insatisfação) foi descartado porque sua presença tornava $\mathrm{O}$ fator semanticamente ininterpretável.

Dada ainda a alta e significante correlação entre eles, havia indícios da existência de um só fator. Por isso, os itens desses dois fatores foram novamente submetidos à análise fatorial (PC) com rotação oblimin e foi solicitada a extração de um único fator. Os resultados dessa análise confirmaram a possibilidade de adotar-se a solução de um único fator, que reuniu os mesmos treze itens dos dois fatores da primeira análise. Este fator explicou $38 \%$ da variância total do conceito de depressão. A análise da fidedignidade, calculada através do alfa de Cronbach, revelou índice altamente satisfatório $(\alpha=0,86)$ (ver Tabela 1$)$.

Tabela 1 - Composição fatorial, comunalidade e composição do fator único e bifatorial

\begin{tabular}{|c|c|c|c|c|c|c|c|}
\hline \multirow[t]{2}{*}{ Itens } & \multicolumn{3}{|c|}{ Fator único: depressão } & \multicolumn{2}{|c|}{$\begin{array}{l}\text { Fator 1: visão } \\
\text { negativa de si } \\
\text { mesmo }\end{array}$} & \multicolumn{2}{|c|}{$\begin{array}{l}\text { Fator 2: função } \\
\text { física }\end{array}$} \\
\hline & Comunalidade & Carga & $\begin{array}{l}\text { Alfa s/ o } \\
\text { item }\end{array}$ & Carga & $\begin{array}{l}\text { Alfa s/ } \\
\text { o item }\end{array}$ & Carga & $\begin{array}{l}\text { Alfa s/ } \\
\text { o item }\end{array}$ \\
\hline Tristeza & 0,47 & 0,63 & 0,90 & 0,35 & 0,80 & & \\
\hline Pessimismo & 0,52 & 0,58 & 0,90 & 0,76 & 0,79 & & \\
\hline Sentimento de fracasso & 0,44 & 0,59 & 0,90 & 0,52 & 0,80 & & \\
\hline Culpa & 0,60 & 0,67 & 0,84 & 0,75 & 0,78 & & \\
\hline Autodepreciação & 0,51 & 0,57 & 0,85 & 0,68 & 0,80 & & \\
\hline Auto-acusação & 0,56 & 0,62 & 0,85 & 0,41 & 0,79 & & \\
\hline Idéias suicidas & 0,55 & 0,58 & 0,85 & 0,54 & 0,80 & & \\
\hline $\begin{array}{l}\text { Mudança na imagem do } \\
\text { corpo }\end{array}$ & 0,55 & 0,72 & 0,84 & 0,52 & 0,79 & & \\
\hline Dificuldade no trabalho & 0,55 & 0,65 & 0,84 & & & $-0,70$ & 0,71 \\
\hline Insônia & 0,53 & 0,58 & 0,85 & & & $-0,73$ & 0,74 \\
\hline Fatigabilidade & 0,63 & 0,72 & 0,84 & & & $-0,72$ & 0,69 \\
\hline Perda de apetite & 0,66 & 0,54 & 0,85 & & & $-0,58$ & 0,74 \\
\hline Perda de libido & 0,43 & 0,50 & 0,85 & & & $-0,65$ & 0,76 \\
\hline \multicolumn{4}{|c|}{$\%$ de variância explicada $=38$} & \multicolumn{2}{|c|}{$\begin{array}{l}\% \text { de variância } \\
\text { explicada }=32,4\end{array}$} & \multicolumn{2}{|c|}{$\begin{array}{l}\% \text { de variância } \\
\text { explicada }=8\end{array}$} \\
\hline
\end{tabular}




\section{Discussão}

Em relação ao BDI, os resultados deste estudo indicaram a possibilidade de uma estrutura fatorial composta de 13 itens descritivos de atitudes e sintomas de depressão. Portanto, houve exclusão de oito dos itens que compunham a escala original (insatisfação, expectativa de punição, retraimento social, indecisão, irritabilidade, perda de peso, choro e preocupação somática). Possivelmente, os itens que se referiam a esses conteúdos não eram importantes para a identificação da depressão nesta amostra.

Alguns estudos que relatam validação fatorial do BDI (Cunha, 2001; Gorenstein \& Andrade, 1998; Schotte e cols., 1997 e Wang e cols., 2005) utilizaram-se de rotação varimax para a extração dos fatores ou componentes. Todavia, em nenhum deles há informações sobre a dependência entre os fatores. Diante da constatação de correlação alta entre os dois fatores identificados neste estudo, optamos pela rotação oblimin, adequada, segundo Tabachnick e Fidell (2001). Dada a ausência de informações dessa natureza nos estudos citados, uma discussão mais profunda acerca da diferença entre os resultados deste e dos estudos anteriormente citados fica inviabilizada. Não se sabe se esses estudos não identificaram correlação entre os fatores ou se isso não foi testado. Caso a interdependência não tenha sido testada, esses estudos falharam ao utilizar a rotação varimax, que pressupõe ortogonalidade entre os fatores (Pasquali, 2006; Tabachnick \& Fidell, 2001). Em contrapartida, Grunebaum e cols. (2005) pressupuseram uma sobreposição entre os componentes da depressão, o que os levou a utilizar uma rotação não-ortogonal, a promax. Contudo, também não relataram nenhum tipo de teste sobre a interdependência entre os fatores que pudesse descartar a utilização da rotação oblimin.

Estudo de Morley e cols. (2002) com pacientes com dor crônica, utilizando rotação oblimin, identificou a existência de três fatores superpostos e inter-relacionados, sendo que os dois últimos concentravam poucos itens com boas cargas, o que foi corroborado pelo estudo aqui relatado. Em uma solução com dois fatores, Morley e cols. (2002) identificaram uma estrutura mais robusta que foi, então, submetida à análise fatorial confirmatória, que reteve 2 fatores com 13 itens: "visão negativa de si mesmo" e "função física" (ver Tabela 2).

A estrutura bifatorial identificada nas primeiras análises do presente estudo assemelhou-se àquela encontrada por Morley e cols. (2002), inclusive quanto ao número de itens retido. Apesar disso, houve diferenças quanto à composição dos fatores e aos itens que permaneceram após as análises (ver Tabela 2).

Dada a boa qualidade das duas soluções fatoriais encontradas no presente estudo, pode-se optar por utilizar o BDI como uma medida bifatorial de depressão, composta por dois agrupamentos de sintomas e atitudes ("visão negativa de si mesmo" e "função física") que explicam $40 \%$ da variância total, ou pela utilização do fator geral de depressão, que explica 38\% da variância total, ambas as soluções com boas cargas fatoriais e índices adequados de fidedignidade. Entretanto, a estrutura bidimensional do BDI, com um fator abrangendo uma dimensão cognitiva e outro, uma dimensão somática é a mais comumente encontrada na literatura, apesar da ocorrência de resultados entre um e sete fatores (Wang e cols., 2005).

A solução de três fatores encontrada por Grunebaum e cols. (2005) com pacientes deprimidos reteve 17 dos 21 itens do BDI, distribuídos da seguinte maneira: F1 - depressão subjetiva; F2 - auto-acusação e F3 - queixas somáticas. Os autores não apresentaram os índices de fidedignidade e os percentuais de explicação da variância total dos fatores. Schotte e cols (1997), que também trabalharam com uma população deprimida, e de forma semelhante à Grunebaum e cols. (2005), obtiveram uma solução com três fatores e mantiveram 17 dos 21 itens (ver Tabela 2).

$\mathrm{Na}$ análise fatorial realizada por Gorenstein e Andrade (1998) com estudantes universitários, as autoras identificaram solução de três fatores com alfa de Cronbach iguais a 0,$76 ; 0,77$ e 0,66 , respectivamente. Na mesma publicação, as autoras identificaram, numa outra amostra de estudantes do ensino fundamental e médio, dois fatores, um deles apresentando alfa de Cronbach de 0,53. Wang e cols. (2005), em uma amostra de chineses que vivem no Brasil, encontraram dois fatores com índices de fidedignidade de 0,79 e 0,67 , respectivamente. Tais trabalhos admitiram um índice de fidedignidade de pelo menos um fator abaixo do mínimo psicometricamente aceitável $(\alpha=0,70)$ segundo Pasquali (2006). Em contraste com tais estudos e confirmando critérios de Pasquali (2006), Schotte e cols. (1997) encontraram uma solução de três fatores, mas, como o terceiro fator possuía $\alpha=0,54$, optaram pela solução bifatorial.

Wang e cols. (2005) comentaram que existiam diversas soluções fatoriais identificadas para o BDI até então. Eles sugerem, como hipóteses para essa diversidade, diferenças metodológicas, como as características populacionais, procedimentos de retenção e extração de fatores, a versão semântica e a abordagem estatística, o que vem ao encontro de nossos questionamentos. Concordamos com os referidos autores e apontamos, a partir do presente estudo, algumas hipóteses para explicar as diferenças aqui encontradas: o método de extração (PC ou PAF), os critérios para a escolha da rotação (varimax, oblimin e promax) na análise fatorial, os critérios para seleção de itens e os índices de fidedignidade admitidos como aceitáveis. 
Tabela 2 - Comparação da composição dos fatores

\begin{tabular}{|c|c|c|c|c|c|c|c|c|}
\hline Item & $\begin{array}{c}\text { Schotte e } \\
\text { cols. } \\
\text { (1997); } \\
\text { pacientes } \\
\text { com } \\
\text { depressão }\end{array}$ & $\begin{array}{c}\text { Morley e } \\
\text { cols. } \\
\text { (2002); } \\
\text { pacientes } \\
\text { com dor } \\
\text { crônica }\end{array}$ & $\begin{array}{c}\text { Grunebaum } \\
\text { e cols. } \\
\text { (2005); } \\
\text { pacientes } \\
\text { deprimidos }\end{array}$ & $\begin{array}{l}\text { Pellegrino- } \\
\text { Rosa } \\
\text { (1997); } \\
\text { idosos } \\
\text { saudáveis }\end{array}$ & $\begin{array}{l}\text { Gorestein \& } \\
\text { Andrade } \\
\text { (1998); } \\
\text { estudantes } \\
\text { universitários }\end{array}$ & $\begin{array}{c}\text { Gorestein } \\
\text { \& Andrade } \\
\text { (1998); } \\
\text { adolescentes }\end{array}$ & $\begin{array}{c}\text { Cunba } \\
\text { (2001); } \\
\text { pacientes } \\
\text { com depen- } \\
\text { dência de } \\
\text { álcool }\end{array}$ & $\begin{array}{c}\text { Gandini, } \\
\text { Martins, } \\
\text { Ribeiro e } \\
\text { Santos; } \\
\text { mulheres } \\
\text { com câncer }\end{array}$ \\
\hline Tristeza & & & f1 & f1 & $\mathrm{f} 2$ & f1 & f1* & f1 \\
\hline Pessimismo & & & f1 & f1 & $\mathrm{f} 2$ & & f3 & f1 \\
\hline $\begin{array}{l}\text { Sentimento de } \\
\text { fracasso }\end{array}$ & f1 & f1 & $\mathrm{f} 2$ & f1 & f1 & f1 & $\mathrm{f} 1 *$ & f1 \\
\hline Culpa & f1 & f1 & $\mathrm{f} 2$ & f1 & f1 & & $\mathrm{f} 1 *$ & f1 \\
\hline $\begin{array}{l}\text { Expectativa de } \\
\text { punição }\end{array}$ & f1 & f1 & f2 & & f1 & f1 & $\mathrm{f} 2 *$ & \\
\hline $\begin{array}{c}\text { Auto- } \\
\text { depreciação }\end{array}$ & f1 & f1 & $\mathrm{f} 2$ & & f1 & f1 & $\mathrm{f} 1 *$ & f1 \\
\hline Auto-acusação & f1 & f1 & $\mathrm{f} 2$ & & f1 & f1 & $\mathrm{f} 1 *$ & f1 \\
\hline Idéias suicidas & f1 & & & & $\mathrm{f} 2$ & f1 & f3 & f1 \\
\hline $\begin{array}{l}\text { Mudança na } \\
\text { imagem } \\
\text { corporal }\end{array}$ & f1 & f1 & f1 & & & f1 & $\mathrm{f} 2 *$ & f1 \\
\hline $\begin{array}{l}\text { Retraimento } \\
\text { social }\end{array}$ & & $\mathrm{f} 2$ & f1 & & $\mathrm{f} 2$ & f1 & $\mathrm{f} 3$ & \\
\hline $\begin{array}{l}\text { Dificuldade no } \\
\text { trabalho }\end{array}$ & $\mathrm{f} 2$ & $\mathrm{f} 2$ & f1 & & & f1 & $\mathrm{f} 2 *$ & $\mathrm{f} 2$ \\
\hline Insônia & $\mathrm{f} 2$ & $\mathrm{f} 2$ & f3 & & f3 & f2 & $\mathrm{f} 2 *$ & $\mathrm{f} 2$ \\
\hline Fatigabilidade & $\mathrm{f} 2$ & $\mathrm{f} 2$ & f1 & & $\mathrm{f} 3$ & & $\mathrm{f} 2 *$ & $\mathrm{f} 2$ \\
\hline Perda de apetite & $\mathrm{f} 2$ & $\mathrm{f} 2$ & $\mathrm{f} 3$ & & f3 & $\mathrm{f} 2$ & $\mathrm{f} 2^{*}$ & $\mathrm{f} 2$ \\
\hline $\begin{array}{l}\text { Preocupação } \\
\text { somática }\end{array}$ & f2 & $\mathrm{f} 2$ & & & f3 & $\mathrm{f} 2$ & $\mathrm{f} 2 *$ & \\
\hline Perda de libido & $\mathrm{f} 2$ & $\mathrm{f} 2$ & f1 & & & & f3 & $\mathrm{f} 2$ \\
\hline Indecisão & f1 & & f1 & & $\mathrm{f} 2$ & $\mathrm{f} 2$ & $\mathrm{f} 3$ & \\
\hline Insatisfação & & & f1 & f1 & $\mathrm{f} 2$ & f1 & $\mathrm{f} 1 *$ & \\
\hline Choro & & & & & $\mathrm{f} 2$ & & $\mathrm{f} 1 *$ & \\
\hline Irritabilidade & f1 & & & & $\mathrm{f} 2$ & $\mathrm{f} 2$ & $\mathrm{f} 1 *$ & \\
\hline Perda de peso & $\mathrm{f} 2$ & & f3 & & & f2 & $\mathrm{f} 2 *$ & \\
\hline
\end{tabular}

* A composição fatorial dos fatores 1 e 2 não está descrita no manual (Cunha, 2001).

A distribuição dos itens aqui apresentada foi estimada a partir dos conteúdos dos itens e da denominação dos fatores feita pela autora naquele manual.

Merecem destaque, ainda, as modificações realizadas neste estudo na amplitude da escala de respostas feitas para aplicar o instrumento. $\mathrm{O}$ instrumento original de Beck possuía escalas de respostas de tamanhos (números de opções de escolha) variadas para alguns itens e, neste estudo, as várias escalas de respostas foram uniformizadas em 4 opções, o que implicou diminuição de sua amplitude em alguns itens. Este procedimento pode ter provocado diminuição na variabilidade das respostas. Todavia, os índices de adequação da amostra para fatoração não indicaram isso $(\mathrm{KMO}=0,90)$.
Apesar de tudo o que foi apontado até então, os resultados indicaram que o BDI possui, para esta amostra, uma estrutura de dois fatores que também pode ser interpretada como um único fator de depressão. Como os diferentes estudos anteriormente relatados demonstram, as investigações sobre o BDI

têm encontrado entre três e sete fatores. O número exato depende do método de extração dos fatores. Estudos usando análise de estrutura latente sugerem que o BDI representa apenas uma única sindrome geral de depressão que reúne três fatores 
inter-relacionados. (Brown, Schulberg \& Madonia, 1995, p. 59)

Estudos de validação de outros instrumentos estrangeiros no Brasil (por ex. em Martins, Guimarães \& Oliveira, 2006, na validação da Escala de Conflitos Intragrupais de Jehn, 1994) revelaram que, muitas vezes, os autores identificaram as mesmas estruturas dos instrumentos originais. Assim, apontar somente as características culturais e educacionais da amostra como responsável pela estrutura fatorial identificada seria simplificar as possíveis causas. Parece que quanto mais sólida é a estrutura do instrumento original, maior a probabilidade de que novos estudos obtenham estruturas semelhantes. Não parece ser este o caso do BDI, sobre o qual os resultados de estudos, nacionais e internacionais, divergem.

$\mathrm{Na}$ verdade, a análise fatorial apontou $\mathrm{O}$ número de "dimensões necessárias para explicar as relações entre as variáveis" (Nunnally \& Bernstein, 1995, p. 503) ou entre os 21 itens do BDI. Identificou, numa primeira análise, chamada de primeira ordem por Nunnally e Bernstein (1995), uma estrutura bifatorial da depressão composta por "visão negativa de si mesmo" e "função física". Observando a correlação entre os dois fatores obtidos, da ordem de $-0,42$, pareceu-nos haver um único fator mais amplo que poderia reunir os dois anteriormente identificados. O fator único, obtido de uma segunda extração que reuniu os itens dos dois fatores da primeira, fator de segunda ordem, segundo Nunnally e Bernstein (1995), embora seja por definição mais abstrato que os de primeira, possibilitou sintetizar a estrutura do construto da depressão no contexto e para a amostra estudada. Isso corrobora posição de Brown e cols. (1995) de que a estrutura do BDI representa uma única síndrome geral de depressão que reúne fatores interdependentes.

\section{Considerações finais e conclusões}

A maioria dos estudos de validação até então publicados utilizaram validades de critério ou convergente e em amostras diferentes desta. Os resultados desta validação fatorial apontaram para a retenção de apenas 13 itens, configurando um instrumento menor do que o original, mas válido e consistente para esta amostra. Apesar disso, este conjunto de itens permitiu identificar a composição fatorial do construto, partindo-se do Inventário de Beck. Há que se avaliar a adequação desta solução fatorial em outras amostras de outras regiões do Brasil para corroborar ou rejeitar a solução identificada neste estudo.
Apesar dos itens retidos diferirem numericamente dos do BDI, não se pode afirmar que esta seria uma forma reduzida daquele. O objetivo deste estudo não foi, como se declarou anteriormente, reduzir o instrumento, mas verificar como ele se estruturava para uma amostra específica. No Brasil não temos outros instrumentos válidos e confiáveis para diagnóstico de depressão nesta população específica. Entretanto, o BDI ainda necessita de novos estudos de validade de construto para amostras específicas e, sobretudo, de padronização e normalização, para que possa servir à interpretação dos resultados de um paciente com câncer comparando-o com outros, já que resultados absolutos nada informam.

No campo aplicado, isso contribuiria para a identificação rápida e eficaz da depressão, o que possibilitaria ao profissional usar menos tempo com um mesmo paciente durante sua avaliação. Com instrumentos válidos e confiáveis, um profissional poderia dispor de mais tempo para o atendimento de outros pacientes e para o suporte de pacientes já diagnosticados. Além disso, convém lembrar que as instituições economizariam financeiramente quanto a horas trabalhadas do profissional para esta fase do trabalho psicológico.

Os resultados encontrados apontaram, também, na direção da necessidade de novos estudos que esclareçam possíveis efeitos dos medicamentos da quimioterapia, visto que a amostra deste estudo foi constituída de $50 \%$ de pacientes em quimioterapia. Outrossim, seria interessante discriminar, em outros trabalhos, pacientes com e sem dor crônica, para a comparação das estruturas de depressão nestas duas condições.

O procedimento de adaptação semântica do instrumento é um aspecto importante, que pode ter influenciado os resultados. Comparando a versão adaptada neste estudo com a versão do BDI atualmente disponível (Cunha, 2001), é possível encontrar muitas semelhanças e algumas diferenças semânticas. Por isso, infere-se a necessidade de uma comparação semântica entre ambas as formas, com teste de clareza de seus conteúdos para confrontá-las e para posterior verificação da equivalência fatorial e diagnóstica de ambas as versões.

\section{Referências}

Beck, A. T. \& Steer, R. A. (1993). Beck Depression Inventory. Manual. San Antonio: Psychology Corporation.

Beck, A. T., Ward, C. H., Mendelson, M., Mock, J. \& Erbaugh, J. (1961). An inventory for measuring depression. Archives of General Psychiatry, 4, 561-571. 
Bennet, D. S., Ambrosini, P. J., Bianchi, M., Barnett, D., Metz, C. \& Babinovich, H. (1997). Relationship of Beck Depression Inventory factors to depression among adolescents. Journal of Affective Disorders, 45(3), 127-134.

Berard, R. M. \& Boermeester, F. (1998). Psychiatric symptomatology in adolescents with cancer. Pediatric Hematology Oncology, 15(3), 211-221.

Brown, C., Schulberg, H. C. \& Madonia, M. J. (1995). Assessing depression in primary care practice with the Beck Depression Inventory and the Hamilton Rating Scale for Depression. Psychological Assessment, 7(1), 59-65.

Chawla, S., Mohanti, B. K., Rakshak, M., Saxena, S., Bahadur, S. \& Rath, G. K. (1999). Temporal assessment of quality of life of head and neck cancer patients receiving radical radiotherapy. Quality of Life Research, 8(1-2), 73-78.

Cunha, J. A. (2001) Manual da versão em português das Escalas Beck. São Paulo: Casa do Psicólogo.

Gandini, R. C., Martins, M. C. F., Ribeiro, M. P. \& Santos, D. T. G. (2001). Validação fatorial do Inventário de Depressão de Beck e outros sintomas psicológicos em mulheres com câncer em geral. (CNPQ Pibic/UFU $\mathrm{n}^{\circ}$ 106/2000). Uberlândia, MG. Universidade Federal de Minas Gerais.

Gorenstein, C. \& Andrade, L (1996). Validation of a Portuguese version of Beck Depression Inventory and the State-Trait Anxiety Inventory in Brazilian subjects. Brazilian Journal of Medical and Biological Research, 29(4), 453-457.

Gorenstein, C. \& Andrade, L. (1998). Inventário de Depressão de Beck: propriedades psicométricas da versão em português. Revista de Psiquiatria Clínica, 25(5), 245-250.

Grunebaum, M. F., Keilp, J., Li, S., Ellis, S. P., Burke, A. K., Oquendo, M. A. \& Mann, J. (2005). Sympton components of standard depression scales and past suicidal behavior. Journal of Affective Disorders, 87(1), 73-82.

Horimoto, C. H., Ayache, D. C. G. \& Souza, J. A. (Orgs.) (2005). Depressão: diagnóstico e tratamento clínico. São Paulo: Roca.

INCA (2005). Instituto Nacional do Câncer. Estimativas da incidência de câncer no Brasil. Disponível em: <www.inca.gov.br>. Acesso em: 1. abr. 2005.

Kelsen, D. P., Portenoy, R. K., Thaler, H. T., Niedzwiecki, D., Passik, S. D., Tao, Y., Banks, W., Brennan, M. F. \& Foley, K. M. (1995). Pain and depression in patients with newly diagnosed pancreas cancer. Journal Clinical Oncology, 13(3), 748755.

Marchioro, G., Azzarello, G., Checchin, F., Perale, M., Segati, R., Sampognaro, E., Rosetti, F., Franchin, A., Pappagallo, G. L. \& Vinante, O. (1996). The impact of a psychological intervention on quality of life in non-metastatic breast cancer. European Journal of Cancer, 32(9), 1612-1615.

Martins, M. C. F., Gandini, R. C. \& Costa, G. N. (2000). Validação fatorial da Escala BSI - Brief Symptom Inventory. Anais do Seminário de Iniciação Cientifica da Universidade Federal de Uberlândia.

Martins, M. C. F., Guimarães, V. F. \& Oliveira, M. C. (2006). Adaptação e validação fatorial da Escala de Conflitos Intragrupais. Anais do II Congresso Brasileiro de Psicologia Organizacional e do Trabalho.

Morley, S., Williams, A. C. C. \& Black, S. (2002) A confirmatory factor analysis of the Beck Depression Inventory in chronic pain. Pain, 99, 289-298.

Nunnaly, J. C. \& Bernstein, I. J. (1995). Teoría Psicométrica. Buenos Aires: McGraw Hill.

Pasquali, L. (2006). Análise fatorial: um manual teóricoprático. Brasília, DF: Laboratório de Pesquisa em Avaliação e Medida.

Pellegrino-Rosa, I. (1997). Variáveis cognitivas e afetivas no envelhecimento saudável (Tese de Doutorado). São Paulo, SP: Universidade Federal de São Paulo.

Pellegrino-Rosa, I., Gandini, R. C., Rosa, J. T., Wutke, E. F. \& Simon, R. (1991). Orientação psicológica de mulheres com câncer de mama sob um ponto de vista sociopsicossomático. Revista Brasileira de Pesquisa em Psicologia, 3(1), 41-52.

Richter, P., Werner, J., Bastine, R., Heerlein, A., Kick, H. \& Sauer, H. (1997). Measuring treatment outcome by the Beck Depression Inventory. Psychopathology, 30(4), 234-240.

Rosa, J. T., Malandrin, M. A., Leite, E. C. \& Silva, J. C. V. V. V. (1986). Um estudo sobre a validação do Inventário de Depressão de Beck. Relato de pesquisa. Anais do Congresso da Sociedade Interamericana de Psicologia, Caracas.

Schotte, C. K. W., Maes, M., Cluydts, R., Doncker, D. \& Cosyns, P. (1997). Construct validity of the Beck Depression Inventory in a depressive population. Journal of Affective Disorders, 49(2), 115-125.

Suarez-Mendoza, A. A., Caballero-Uribe, C. V., Ortega-Soto, H. A. \& Marquez-Marin, M. (1997). Measurement of depression in Mexican patients 
with rheumatoid arthritis: validity of the Beck Depression Inventory. Arthritis Care and Research, 10(3), 194-199.

Tabachnick, B. G. \& Fidell, L. S. (2001). Using multivariate statistics. New York: Harper and Row.

Wang, Y.-P., Andrade, L. H. \& Gorenstein, C. (2005). Validation of the Beck Depression Inventory for a portuguese-speaking chinese community in Brazil.
Brazilian Journal of Medical and Biological Research, 38(3), 399-408.

Recebido em novembro de 2005

Reformulado em novembro de 2006

Aprovado em fevereiro de 2007

Sobre as autoras:

Rita de Cássia Gandini é doutora em Psicologia Clínica pelo IP-USP/SP, com Pós-Doutorado em Psicooncologia pela Universidade de Ferrara - Itália, mestre em Psicologia da Saúde pela UMESP/SBC, professora da Pós-Graduação e da Graduação em Psicologia do Instituto de Psicologia, coordenadora do Programa de Psicooncologia na Mastologia da Universidade Federal de Uberlândia e pós-doutoranda da Escola de Enfermagem de Ribeirão Preto USP (de 15/4/2006 a 14/4/2007).

Maria do Carmo Fernandes Martins é doutora em Psicologia pela Universidade de Brasília, professora da Universidade Federal de Uberlândia e coordenadora do Programa de Pós-Graduação em Psicologia. Atua na área de Comportamento Organizacional, investigando temas como clima, poder e comprometimentos organizacionais, avaliação de treinamento, efetividade e satisfação no trabalho. É autora de várias escalas de medida, de artigos e capítulos de livros.

Marjorie de Paula Ribeiro é graduada pela Unversidade Federal de Uberlândia, bolsista de Iniciação Científica do CNPq processo PIBIC/UFU no 106/2000, Especialização em Psicodrama Terapêutico, psicóloga do Instituto de Previdência e Assistência dos Servidores do Estado de Goiás - IPAGO, trabalhando no Programa IPASGO Domiciliar.

Daniela Torres Gonçalves Santos é graduada pela Universidade Federal de Uberlândia, com co-participação na Iniciação Científica do CNPq processo PIBIC/UFU no 106/2000, atualmente psicóloga judicial do Tribunal de Justiça de Minas Gerais. 
